\title{
Determinants and Clinical Outcomes of Extended Dual Antiplatelet Therapy over 3 Years after Drug-Eluting Stent Implantation: A Retrospective Analysis
}

\author{
Oh-Hyun Lee ${ }^{1,2}$, Byeong-Keuk Kim¹, Sung-Jin Hong', Seunghwan Kim³ , Chul-Min Ahn' , Dong-Ho Shin ${ }^{1}$, \\ Jung-Sun Kim ${ }^{1}$, Tae Soo Kang ${ }^{4}$, Young-Guk Ko ${ }^{1}$, Donghoon Choi ${ }^{1,2}$, Myeong-Ki Hong ${ }^{1}$, and Yangsoo Jang \\ ${ }^{1}$ Division of Cardiology, Severance Cardiovascular Hospital, Yonsei University College of Medicine, Seoul; \\ ${ }^{2}$ Division of Cardiology, Department of Internal Medicine, Yonsei University College of Medicine and Cardiovascular Center, \\ Yongin Severance Hospital, Yongin; \\ ${ }^{3}$ Division of Cardiology, Department of Internal Medicine, Haeundae Paik Hospital, Inje University College of Medicine, Busan; \\ ${ }^{4}$ Division of Cardiovascular Medicine, Department of Internal Medicine, Dankook University Hospital, Dankook University School of Medicine, \\ Cheonan, Korea.
}

Purpose: Although current guidelines recommend the administration of dual antiplatelet therapy (DAPT) for up to 12 months after the implantation of a drug-eluting stent (DES), extended DAPT is frequently used in real-world practice.

Materials and Methods: From the Korean Multicenter Angioplasty Team registry, we identified a total of 1414 patients who used DAPT for $>3$ years after DES implantation (extended-DAPT group) and conducted a landmark analysis at 36 months after the index procedure. We evaluated the determinants for and long-term outcomes of extended DAPT and compared the occurrence of major adverse cardiovascular and cerebrovascular events (MACCE), defined as the composite of all-cause death, myocardial infarction, stent thrombosis, and stroke, between the extended-DAPT group and the guideline-DAPT group [DAPT $<1$ year after DES implantation ( $\mathrm{n}=1273)]$.

Results: Multivariate analysis indicated the occurrence of acute coronary syndrome as the most significant clinical determinant of the use of extended DAPT. Bifurcation, stent diameter $\leq 3.0 \mathrm{~mm}$, total stented length $\geq 28 \mathrm{~mm}$, and use of first-generation DESs were also significant angiographic and procedural determinants. MACCE rates were similar between the extended-DAPT group and the guideline-DAPT group in crude analysis [hazard ratio (HR), 1.08; 95\% confidence interval (CI), 0.69-1.68; $p=0.739$ ] and after propensity matching (HR, 1.22; 95\% CI, $0.72-2.07 ; p=0.453$ ). Major bleeding rates were comparable between the two groups.

Conclusion: In patients undergoing percutaneous coronary intervention, indefinite use of DAPT does not show superior outcomes to those of guideline-DAPT. Major bleeding rates are also similar.

Key Words: Antiplatelet therapy, drug-eluting stent, percutaneous coronary intervention

Received: January 7, 2020 Revised: May 13, 2020

Accepted: May 13, 2020

Corresponding author: Byeong-Keuk Kim, MD, PhD, Division of Cardiology, Severance Cardiovascular Center, Yonsei University College of Medicine, 50-1 Yonseiro, Seodaemun-gu, Seoul 03722, Korea.

Tel: 82-2-2228-8468, Fax: 82-2-393-2041, E-mail: KIMBK@yuhs.ac

-The authors have no potential conflicts of interest to disclose

(C) Copyright: Yonsei University College of Medicine 2020

This is an Open Access article distributed under the terms of the Creative Commons Attribution Non-Commercial License (https://creativecommons.org/licenses/ by-nc/4.0) which permits unrestricted non-commercial use, distribution, and reproduction in any medium, provided the original work is properly cited.

\section{INTRODUCTION}

The duration of dual antiplatelet therapy (DAPT) in the drugeluting stent (DES) era remains controversial. ${ }^{1}$ Current clinical practice guidelines recommend the use of DAPT for 6 to 12 months after DES implantation. ${ }^{2}$ However, in real-world practice, the use of DAPT for more than 12 months, the use of extended DAPT for more than 3 years, and nearly indefinite use of DAPT are common for various reasons. A recent large randomized controlled trial indicated that the use of DAPT be- 
yond 1 year after DES implantation significantly reduced the risk of stent thrombosis (ST) and major adverse events, but increased the risk of bleeding, which was associated with an increase in mortality. ${ }^{3}$ Therefore, we sought to investigate the major clinical, angiographic, and procedural determinants for extended-DAPT use (DAPT $>3$ years), and to compare the long-term clinical outcomes between patients who used DAPT for more than 3 years and those who used DAPT for up to 12 months.

\section{MATERIALS AND METHODS}

Between April 2003 and March 2012, patients who underwent percutaneous coronary intervention (PCI) using DESs and met the study criteria were enrolled in the Korean Multicenter Angioplasty Team registry. ${ }^{4} \mathrm{~A}$ total of a 4081 patients were identified for analysis after excluding patients with the following conditions: 1) major adverse events such as death, myocardial infarction (MI), ST, repeated revascularization, stroke, or major bleeding; 2) clinical follow-up duration of $<3$ years; and 3 ) unclear DAPT duration (Fig. 1). Patients were classified into three groups according to DAPT duration after DES implantation as follows: patients who used DAPT for more than 3 years (extended-DAPT group; $\mathrm{n}=1414$ ); patients who used DAPT for 1-3 years (intermediate-DAPT; $n=1394$ ); and patients who discontinued DAPT and switched to single antiplatelet therapy within 12 months according to clinical guidelines (guidelineDAPT group; $n=1273$ ). To evaluate the major determinants for long-term outcomes of extended DAPT use, various baseline clinical, angiographic, and procedural characteristics and clinical outcomes of the extended-DAPT group were compared to those of the guideline-DAPT group. To compare the clinical outcomes, a landmark analysis was performed 3 years after the index procedure. In this study, DAPT was defined as the combination of aspirin and clopidogrel, and patients who received other P2Y12 inhibitors were not included. The Institutional Review Board or ethics committee approved the study protocol (4-2018-0759), and all patients provided written informed consent before participating in the study.

Data were recorded using standard case report forms, and procedural angiographies were forwarded to the coordinating center. Baseline clinical and angiographic data were collected for all patients, including age, sex, traditional coronary risk factors, past medical history, and clinical presentation.

Follow-up included visits to the outpatient clinic at 30 days and 3 months after the procedure and every 3 to 6 months thereafter. Patients who were lost to regular clinical follow-up were contacted by telephone and asked about their clinical status.

The primary endpoints were 1) major clinical, angiographic, and procedural determinants for extended-DAPT use (DAPT $>3$ years) and 2) the occurrence of major adverse cardiovascular and cerebrovascular events (MACCE), defined as the composite of all-cause death, MI, ST, and stroke. The secondary endpoints were 1) net adverse clinical events (NACE), defined as the composite of all-cause death, MI, ST, stroke, and major bleeding; 2) the composite of cardiac death, MI, and ST; and 3) individual components of MACCE and NACE.

All events were defined according to the Academic Research Consortium and an expert consensus document that defined the third universal definition for MI. ${ }^{5,6}$ Deaths were classified as being due to cardiac or noncardiac causes. ${ }^{5} \mathrm{MI}$ was defined as a creatine kinase myocardial band fraction elevation above the upper limit of normal (ULN) or troponin T/I $>99$ th percentile of the ULN, with concomitant ischemic symptoms, electrocardiographic findings, or abnormal imaging findings indicative of myocardial ischemia unrelated to an interventional procedure. ${ }^{5,6}$ ST was defined as definite or probable ST. ${ }^{5}$ Stroke was defined as brain cell death caused by ischemia based on pathological, imaging, or other objective evidence of cerebral focal ischemic injury in a defined vascular distribution or clinical evidence of cerebral focal ischemic injury based on symptoms persisting $\geq 24$ hours or until death; other etiologies were excluded. ${ }^{7}$ Strokes were determined to be either hemorrhagic or ischemic using imaging studies. Major bleeding was defined as type 3 or 5 according to the Bleeding Academic Research Consortium criteria. ${ }^{8}$ Congestive heart failure was defined as left ventricle ejection fraction $<40 \% .{ }^{9}$ Bifurcation lesions were defined as Medina classification (1.1.1), (1.0.1), or (0.1.1) lesions. ${ }^{10}$ The DAPT scoring system assigned 1 point each for MI at presentation, prior MI or PCI, diabetes, stent diameter $<3 \mathrm{~mm}$, cigarette smoking, and paclitaxel-eluting stent; 2 points each for the history of congestive heart failure/low ejection fraction $(<30 \%)$ and vein graft intervention; -1 point for age $65-75$ years; and -2 points for age $\geq 75$ years. ${ }^{11}$

Continuous variables are expressed as a mean \pm standard deviation, and categorical data are presented as frequencies. For group comparisons, Pearson's chi-square test, Fisher's chi-exact test, or Wilcoxon rank-sum test were used, as appropriate. Timeto-event data are presented using Kaplan-Meier estimates. The rates for each event during the follow-up period were analyzed using Kaplan-Meier method. Log-rank test was used to compare survival rates. Hazard ratios (HR) with 95\% confidence intervals (CI) were estimated from a Cox proportional hazards model. To identify the determinants for the use of extendedDAPT, we performed univariate and multivariate analyses. Any variable with $p$ value $<0.10$ in univariate analysis was included in the multivariate models. To reduce bias between the two groups in comparison, propensity score matching was used. Propensity score adjusting was used in the logistic regression models, with DAPT continuation at 36 months used as the dependent variable along with 27 independent variables. A two-sided $p$ value $<0.05$ was considered statistically significant. Statistical analyses were performed using SPSS statistical software (SPSS version 23.0 for Windows, IBM Corp., Armonk, NY, USA). 


\section{RESULTS}

Among the eligible patients $(n=4081), 1414$ (34.6\%) were included in the extended-DAPT group and 1273 were included in the guideline-DAPT group (Fig. 1). The baseline clinical characteristics of both groups are presented in Table 1. Compared to the guideline-DAPT group, the extended-DAPT group had higher incidences of prior MI, PCI, and bypass surgery; and acute coronary syndrome (ACS); and a lower left ventricular ejection fraction.

Angiographic and procedural characteristics are provided in Table 2. Compared to the guideline-DAPT group, the extended-DAPT group had higher proportions of individuals with lesions with multi-vessel disease, in-stent restenosis, bifurcation, and number of lesions treated $\geq 2$. The extended-DAPT group had a greater number of stents implanted, longer- and smaller-sized stents used, and a more frequent use of first-generation DESs compared to the guideline-DAPT group.

DAPT scores were higher in the extended-DAPT group than in the guideline-DAPT group $(p<0.01)$, and the proportion of patients with DAPT scores greater than 2 points was higher in the extended-DAPT group (50.3\% vs. $29.3 \%, p<0.01$ ) (Supplementary Table 1 , only online).

In multivariate analyses for the determinants of extended use of DAPT, ACS presentation was the most significant clinical

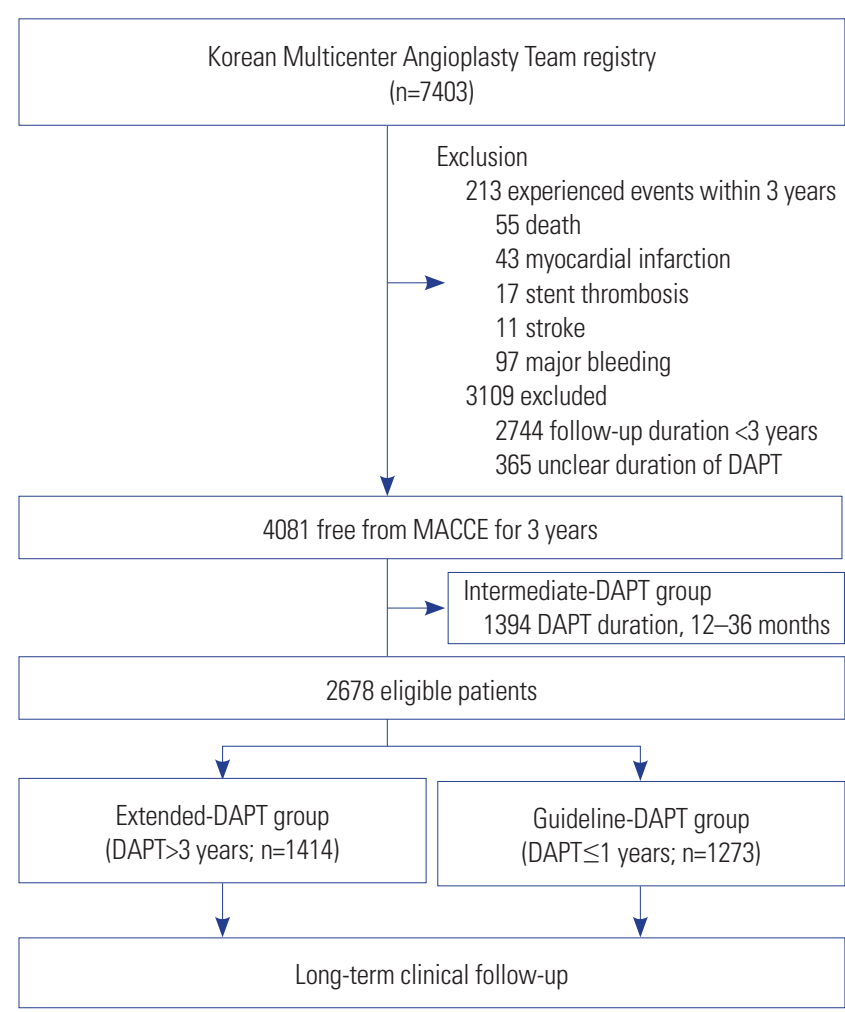

Fig. 1. Study at a glance. DAPT, dual antiplatelet therapy; MACCE, major adverse cardiovascular and cerebrovascular events.

Table 1. Clinical Characteristics

\begin{tabular}{|c|c|c|c|}
\hline Characteristics & Extended-DAPT (n=1414) & Guideline-DAPT ( $\mathrm{n}=1273$ ) & $p$ value \\
\hline Age (yr) & $62.8 \pm 9.8$ & $62.7 \pm 9.9$ & 0.809 \\
\hline Male & $927(66)$ & $858(67)$ & 0.456 \\
\hline Body mass index $\left(\mathrm{kg} / \mathrm{m}^{2}\right)$ & $24.8 \pm 3.0$ & $24.8 \pm 2.9$ & 0.950 \\
\hline Current smoker & $308(22)$ & $301(24)$ & 0.249 \\
\hline Dyslipidemia or statin use before procedure & $1160(82)$ & $1057(83)$ & 0.498 \\
\hline Hypertension & $879(62)$ & $808(64)$ & 0.484 \\
\hline Diabetes mellitus & $447(32)$ & $387(30)$ & 0.498 \\
\hline Chronic kidney disease & $39(3)$ & $23(2)$ & 0.101 \\
\hline Congestive heart failure & $38(3)$ & $27(2)$ & 0.340 \\
\hline Left ventricular ejection fraction (\%) & $57.8 \pm 13.4$ & $61.4 \pm 12.3$ & $<0.001$ \\
\hline Prior stroke & $116(8)$ & $107(8)$ & 0.850 \\
\hline Prior myocardial infarction & $157(11)$ & $71(6)$ & $<0.001$ \\
\hline Previous percutaneous coronary intervention & $265(19)$ & $136(11)$ & $<0.001$ \\
\hline Prior bypass surgery & $59(4)$ & $35(3)$ & 0.045 \\
\hline Clinical presentation & & & $<0.001$ \\
\hline Acute coronary syndrome & $652(46)$ & $492(39)$ & \\
\hline Acute myocardial infarction & $301(21)$ & $249(20)$ & \\
\hline Unstable angina & $351(25)$ & $243(19)$ & \\
\hline Stable angina & $762(54)$ & $781(61)$ & \\
\hline \multicolumn{4}{|l|}{ Medications at discharge } \\
\hline$\beta$-blockers & $849(60)$ & $741(58)$ & 0.330 \\
\hline Angiotensin-converting enzyme or angiotensin-receptor blockers & $789(56)$ & $724(57)$ & 0.583 \\
\hline Statin & $1376(97)$ & $1235(97)$ & 0.642 \\
\hline
\end{tabular}

DAPT, dual antiplatelet therapy.

Data are presented as the mean \pm SD or percentage $(\%)$. 
Table 2. Angiographic and Procedural Characteristics

\begin{tabular}{|c|c|c|c|}
\hline Characteristics & $\begin{array}{c}\text { Extended- } \\
\text { DAPT } \\
(n=1414)\end{array}$ & $\begin{array}{c}\text { Guideline- } \\
\text { DAPT } \\
(n=1273)\end{array}$ & $p$ value \\
\hline Multi-vessel (2- or 3-vessel) disease & $980(69)$ & $742(58)$ & $<0.001$ \\
\hline Treated vessel & & & 0.596 \\
\hline Left anterior descending & $813(58)$ & $761(60)$ & \\
\hline Left circumflex & $227(16)$ & $184(15)$ & \\
\hline Right coronary & $365(26)$ & $320(25)$ & \\
\hline Graft & $9(1)$ & $8(1)$ & \\
\hline Left main artery involvement & $121(9)$ & $112(9)$ & 0.825 \\
\hline Chronic total occlusion & $71(6)$ & $57(5)$ & 0.243 \\
\hline In-stent restenosis & $69(9)$ & $30(3)$ & $<0.001$ \\
\hline Bifurcation & $385(50)$ & $366(36)$ & $<0.001$ \\
\hline Calcification (moderate or severe) & $131(11)$ & $127(11)$ & 0.853 \\
\hline Number of lesions treated $\geq 2$ & $549(39)$ & $345(27)$ & $<0.001$ \\
\hline Total number of stents implanted & $1.6 \pm 0.7$ & $1.5 \pm 0.6$ & $<0.001$ \\
\hline Total stent length & $40.5 \pm 21.3$ & $32.8 \pm 17.6$ & $<0.001$ \\
\hline Total stent length $\geq 28 \mathrm{~mm}$ & $968(69)$ & $675(53)$ & $<0.001$ \\
\hline Stent diameter & $3.0 \pm 0.4$ & $3.1 \pm 0.4$ & $<0.001$ \\
\hline Stent diameter $\leq 3.0 \mathrm{~mm}$ & $1106(78)$ & $571(45)$ & $<0.001$ \\
\hline Type of drug-eluting stents & & & $<0.001$ \\
\hline First-generation & $1078(76)$ & $313(25)$ & $<0.001$ \\
\hline Sirolimus-eluting & 762 & 256 & \\
\hline Paclitaxel-eluting & 316 & 57 & \\
\hline Second-generation & $269(19)$ & $932(73)$ & \\
\hline Everolimus-eluting & 5 & 288 & \\
\hline Zotarolimus-eluting & 199 & 379 & \\
\hline Biolimus-eluting & 15 & 265 & \\
\hline $\begin{array}{l}\text { Mixed use of the first- and } \\
\text { second-generation }\end{array}$ & $67(5)$ & $28(2)$ & \\
\hline Glycoprotein $\left\|_{B} /\right\| \|_{A}$ blocker use & $21(4)$ & $20(3)$ & 0.391 \\
\hline
\end{tabular}

DAPT, dual antiplatelet therapy.

Data are presented as the mean \pm SD or percentage $(\%)$.

determinant. Of the angiographic and procedural variables, bifurcation, total stent length $\geq 28 \mathrm{~mm}$, stent diameter $\leq 3.0$ $\mathrm{mm}$, and use of first-generation DESs were significant determining factors for the extended use of DAPT (Table 3 ).

During follow-up (median duration, 120 months), there were 79 MACCEs (2.9\%; 36 all-cause deaths, 29 MI, 6 ST, 22 strokes) after DES implantation, and no significant difference was noted between the extended-DAPT and guideline-DAPT groups in both the crude- and propensity-matched analyses (Table 4 and Fig. 2). The rates of MACCE and NACE were not significantly different between the two groups in either the crudeor propensity-matched analyses. The rates of composite allcause death, MI, and ST were also not significantly different between the two groups in both the crude- and propensitymatched analyses. The rates of major bleeding were similar between the two groups ( $p=0.185)$. In propensity-matched analyses, the extended-DAPT group showed a trend toward having a higher major bleeding rate compared to the guideline-DAPT group, although the difference was not statistically significant $(p=0.070)$

We also performed subgroup analyses while focusing on the major determining factors for extended-DAPT (Fig. 3). In the subgroup analyses of composite of all-cause death, MI, and ST, a significant interaction was not noted. In multivariate analysis for the composite of all-cause death, MI, and ST, age (per year) and total stent length $\geq 28 \mathrm{~mm}$ were significant risk factors (Supplementary Table 2, only online).

\section{DISCUSSION}

The principal findings of the current study are as follows: 1 ) In real-world practice, extended DAPT is frequently used in patients without MACCE and with DES implantation (approximately $1 / 3$ of patients). 2) Significant determinants for the extended use of DAPT for more than 3 years were ACS, bifurcation, small diameter and long length of DES, and use of first-generation DESs. 3) In patients undergoing PCI, the outcomes after extended-DAPT, defined as indefinite use of DAPT with aspirin and clopidogrel, did not show superiority over those after the guideline-DAPT, and major bleeding rates were similar. 4) In patients treated with first-generation DESs, extended use of DAPT was significantly associated with lower MACCE rates compared to the use of guideline-DAPT.

Antiplatelet treatment is a cornerstone in the management of patients with DES implantation for preventing ST. ${ }^{2}$ However, even with the designated duration recommended in current guidelines, the optimal DAPT duration after DES implantation still remains debatable. ${ }^{3,12,13}$ Both prolonged DAPT for longer than 1 year and short-term DAPT for 3-6 months have been proposed. A recent randomized DAPT trial demonstrated that the use of DAPT beyond 1 year after the placement of a DES significantly reduced the risks of ST ( $1.4 \%$ vs. $0.4 \%)$ and MACCE (5.9\% vs. $4.3 \%$ ) compared to using aspirin therapy alone. A previous meta-analysis also indicated that DAPT for 1 year after DES implantation reduced the occurrence of MI and ST, but increased mortality due to an increased risk of non-cardiovascular mortality. ${ }^{14}$ In the current real-world registry study, $34.6 \%$ of patients who had not experienced MACCE for 3 years still received DAPT at 3 years. Similarly, the patterns of nonadherence to antiplatelet regimens in stented patients (PARIS) registry investigating DAPT patterns in postPCI patients indicated that $42.0 \%$ of patients were still receiving DAPT at 2 years. ${ }^{15}$ Thus, there appear to be some conflict between the guideline recommendations and the actual use in recent clinical trials. In addition, the incidence and determinants for the extended use of DAPT have not been clearly established in real-world practice.

Many scoring systems have been suggested for determining the duration of DAPT..$^{11,16,17}$ In particular, DAPT, PARIS, and the Predicting Bleeding Complications In Patients Undergoing 
Oh-Hyun Lee, et al.

Table 3. Univariate and Multivariate Analyses of Determinants for the Extended Use of DAPT

\begin{tabular}{|c|c|c|c|c|}
\hline \multirow{2}{*}{ Variables } & \multicolumn{2}{|c|}{ Univariate analysis } & \multicolumn{2}{|c|}{ Multivariate analysis* } \\
\hline & OR $(95 \% \mathrm{Cl})$ & $p$ value & OR $(95 \% \mathrm{Cl})$ & $p$ value \\
\hline \multicolumn{5}{|l|}{ Clinical variables } \\
\hline Age (yr) & $1.00(0.99-1.01)$ & 0.809 & $1.00(0.98-1.01)$ & 0.462 \\
\hline Body mass index $\left(\mathrm{kg} / \mathrm{m}^{2}\right)$ & $1.01(0.97-1.03)$ & 0.950 & & \\
\hline Male & $1.06(0.90-1.25)$ & 0.456 & $1.26(0.95-1.67)$ & 0.114 \\
\hline Hypertension & $0.95(0.81-1.11)$ & 0.484 & & \\
\hline Diabetes mellitus & $1.06(0.90-1.25)$ & 0.498 & & \\
\hline Chronic kidney disease & $1.54(0.92-2.60)$ & 0.103 & & \\
\hline Current smoker & $0.90(0.75-1.08)$ & 0.250 & & \\
\hline Acute coronary syndrome presentation & $1.38(1.18-1.61)$ & $<0.001$ & $1.37(1.06-1.79)$ & 0.018 \\
\hline Prior percutaneous coronary intervention & $1.93(1.54-2.41)$ & $<0.001$ & $1.43(0.92-2.21)$ & 0.113 \\
\hline Prior bypass surgery & $1.54(1.01-2.36)$ & 0.047 & $1.22(0.59-2.53)$ & 0.598 \\
\hline Prior myocardial infarction & $2.12(1.58-2.83)$ & $<0.001$ & $1.55(0.96-2.49)$ & 0.072 \\
\hline Prior heart failure & $1.27(0.77-2.10)$ & 0.341 & & \\
\hline Prior stroke & $0.97(0.74-1.28)$ & 0.850 & & \\
\hline \multicolumn{5}{|l|}{ Angiographic variables } \\
\hline Treated lesion number $\geq 2$ & $1.71(1.45-2.01)$ & $<0.001$ & $0.91(0.65-1.30)$ & 0.613 \\
\hline Multivessel disease & $1.62(1.38-1.89)$ & $<0.001$ & $1.16(0.85-1.58)$ & 0.345 \\
\hline Left main involvement & $0.97(0.74-1.27)$ & 0.825 & & \\
\hline In-stent restenosis & $3.38(2.18-5.24)$ & $<0.010$ & $2.04(0.99-4.20)$ & 0.053 \\
\hline Bifurcation & $1.78(1.47-2.15)$ & $<0.001$ & $1.55(1.19-2.02)$ & 0.001 \\
\hline Chronic total occlusion & $1.24(0.87-1.77)$ & 0.244 & & \\
\hline \multicolumn{5}{|l|}{ Procedural variables } \\
\hline Total stent length $\geq 28 \mathrm{~mm}$ & $1.92(1.64-2.25)$ & $<0.001$ & $1.54(1.12-2.12)$ & 0.007 \\
\hline Stent diameter $\leq 3.0 \mathrm{~mm}$ & $4.42(3.73-5.22)$ & $<0.001$ & $3.33(2.51-4.43)$ & $<0.001$ \\
\hline First-generation drug-eluting stents & $11.91(9.93-14.29)$ & $<0.001$ & 11.43 (8.78-14.87) & $<0.001$ \\
\hline
\end{tabular}

DAPT, dual antiplatelet therapy; $\mathrm{OR}$, odds ratio; $\mathrm{Cl}$, confidence interval.

${ }^{*}$ Any variable with $p<0.10$ in univariate analysis was included in the multivariate models.

Stent Implantation and Subsequent Dual Antiplatelet Therapy (PRECISE-DAPT) score have focused on clinical determinants for the optimal duration of DAPT. In the current study, the proportion of patients with a DAPT score of more than 2 points was significantly higher in the extended-DAPT group, and the initial clinical presentation of ACS was the most significant clinical factor that determined the duration of DAPT. Indeed, previous studies have reported that the use of extended DAPT in ACS patients reduces recurrent ischemic events, and that a significant proportion of patients experience adverse cardiac events beyond the first year after ACS. ${ }^{18}$

Lesion- or procedural-complexity could be considered when determining DAPT duration. However, these are not considered in the current scoring systems that are used to determine DAPT duration. In contrast, lesion- and procedural-complexity were identified as major determinants for the use of extended DAPT in this study. Stent-related predictors were also significant determining factors for DAPT duration. In the current study, the total stented length of $\geq 28 \mathrm{~mm}$, minimal stent diameter of $\leq 3.0 \mathrm{~mm}$, and the use of first-generation DES were major determining factors. Previous meta-analyses indicated that the most significant predictors of ST include longer stent length [odds ratio (OR), 1.3; 95\% CI, 1.2-1.5, per $10 \mathrm{~mm}$ ] and final minimal lumen diameter with stent (OR, 04; 95\% CI, 0.20.7 , per $1 \mathrm{~mm}) .{ }^{19}$ Another study on the etiology of very-late ST also indicated that patients with very-late ST had longer lesions and stents. ${ }^{20}$ Therefore, physicians tend to use extended DAPT. A significant angiographic predictor in this study was the occurrence of a bifurcation lesion. This may have been related to the increasing concerns caused by a higher risk of ST after the use of the two-stent technique or malapposed metal burden in the jailed side branch from the use of a cross-over single stent. A previous study reported that the risk of allcause death or MI was significantly lower in the $\geq 12$-month DAPT group than in the $<12$-month DAPT group after PCI for bifurcation lesions using DES. ${ }^{21}$ 
Table 4. Clinical Outcomes at 10 Years (Median Follow-Up Duration)

\begin{tabular}{|c|c|c|c|c|}
\hline Crude analysis & Extended-DAPT ( $n=1414)$ & Guideline-DAPT ( $n=1273$ ) & HR (95\% Cl) & $p$ value \\
\hline Follow-up duration (month) & $111.11 \pm 18.8$ & $103.0 \pm 18.0$ & & \\
\hline MACCE (all-cause death, MI, ST, and stroke) & $44(3.3)$ & $35(3.2)$ & $1.08(0.69-1.68)$ & 0.739 \\
\hline NACE (all-cause death, MI, ST, stroke, and major bleeding) & $60(4.7)$ & $43(4.4)$ & $1.10(0.74-1.64)$ & 0.629 \\
\hline Composite of all-cause death, MI, and ST & $32(2.4)$ & $30(2.8)$ & $0.91(0.55-1.49)$ & 0.700 \\
\hline Death & $22(1.7)$ & $14(1.4)$ & $1.31(0.67-2.56)$ & 0.434 \\
\hline Cardiovascular death & $2(0.1)$ & $4(0.4)$ & $0.41(0.07-2.24)$ & 0.303 \\
\hline Non-cardiovascular death & $20(1.5)$ & $10(1.0)$ & $1.67(0.78-3.58)$ & 0.186 \\
\hline $\mathrm{Ml}$ & $14(1.1)$ & $15(1.3)$ & $0.80(0.39-1.66)$ & 0.553 \\
\hline ST & $3(0.2)$ & $3(0.3)$ & $0.84(0.17-4.19)$ & 0.832 \\
\hline Total stroke & $13(1.0)$ & $9(0.8)$ & $1.25(0.53-2.92)$ & 0.611 \\
\hline Ischemic stroke & $11(0.8)$ & $8(0.7)$ & $1.18(0.47-2.94)$ & 0.726 \\
\hline Hemorrhagic stoke & $2(0.1)$ & $3(0.3)$ & $0.60(0.10-3.56)$ & 0.569 \\
\hline Major bleeding & $19(1.5)$ & $9(1.2)$ & $1.71(0.77-3.80)$ & 0.185 \\
\hline Propensity score matching analysis & Extended DAPT ( $n=677$ ) & Guideline DAPT ( $n=1149$ ) & HR (95\% Cl) & $p$ value \\
\hline Follow-up duration, months & $108.4 \pm 21.7$ & $102.8 \pm 18.4$ & & \\
\hline MACCE (all-cause death, MI, ST, and stroke) & $24(3.8)$ & $33(3.2)$ & $1.22(0.72-2.07)$ & 0.453 \\
\hline NACE (all-cause death, MI, ST, stroke, and major bleeding) & $36(6.1)$ & $39(4.2)$ & $1.43(0.90-2.25)$ & 0.128 \\
\hline Composite of all-cause death, MI, and ST & $16(2.5)$ & $28(2.8)$ & $0.96(0.52-1.78)$ & 0.895 \\
\hline Death & $12(1.9)$ & $13(1.3)$ & $1.51(0.69-3.31)$ & 0.306 \\
\hline Cardiovascular death & $1(0.2)$ & $4(0.5)$ & $0.39(0.04-3.52)$ & 0.402 \\
\hline Non-cardiovascular death & $11(1.8)$ & $9(0.8)$ & $2.02(0.84-4.88)$ & 0.119 \\
\hline $\mathrm{Ml}$ & $7(1.1)$ & $14(1.4)$ & $0.84(0.34-2.09)$ & 0.713 \\
\hline ST & $2(0.3)$ & $3(0.3)$ & $1.07(0.18-6.42)$ & 0.944 \\
\hline Total stroke & $8(1.3)$ & $8(0.8)$ & $1.64(0.61-4.39)$ & 0.324 \\
\hline Ischemic stroke & $8(1.3)$ & $8(0.8)$ & $1.64(0.61-4.39)$ & 0.324 \\
\hline Hemorrhagic stoke & 0 & 0 & - & - \\
\hline Major bleeding & $12(2.1)$ & $8(1.2)$ & $2.29(0.93-5.63)$ & 0.070 \\
\hline
\end{tabular}

DAPT, dual antiplatelet therapy; HR, hazard ratio; Cl, confidence interval; MACCE, major adverse cardiovascular and cerebrovascular events; NACE, net adverse clinical events; MI, myocardial infarction; ST, stent thrombosis.

Data are presented as the mean \pm SD or number (\%) and percentages, along with Kaplan-Meier estimates.

A

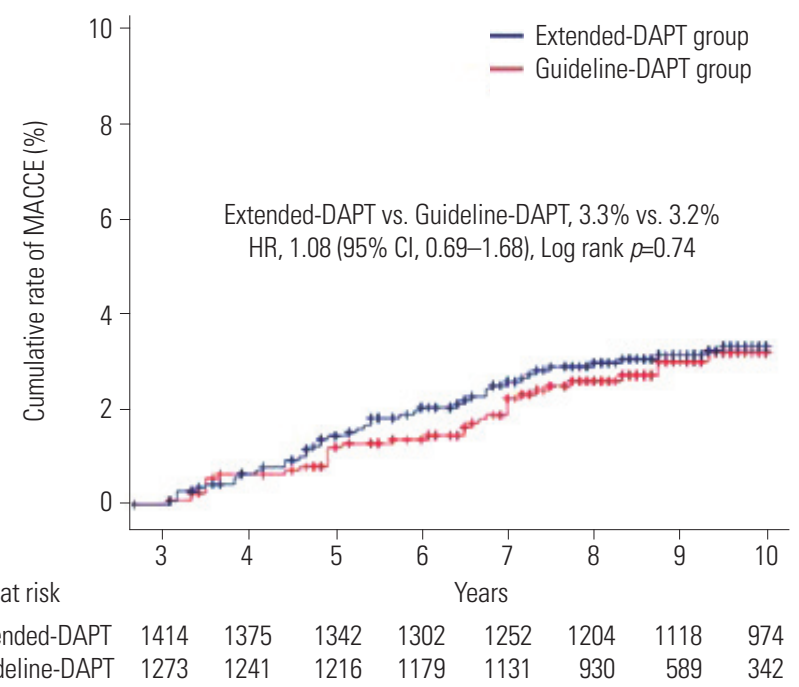

B

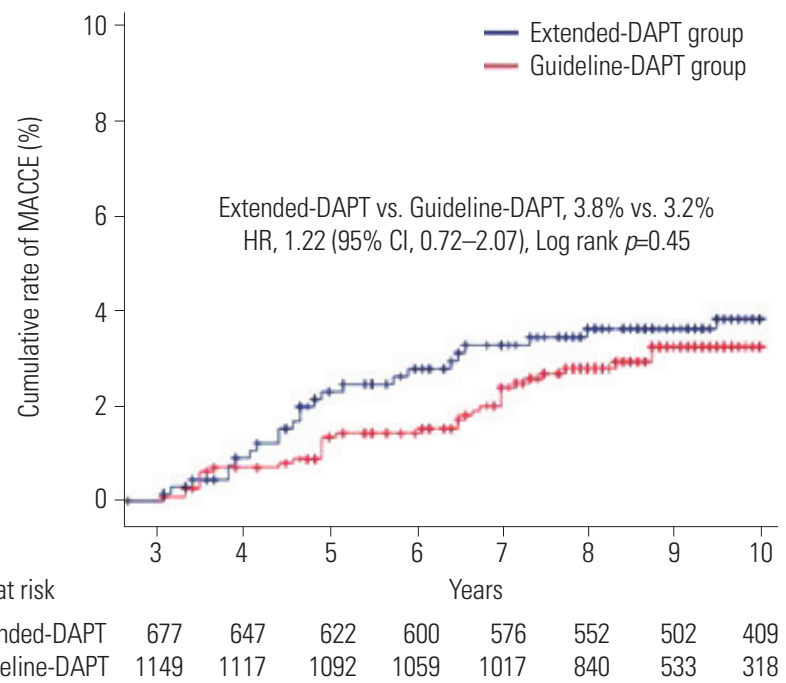

Fig. 2. Cumulative MACCE rates according to Kaplan-Meier analyses. (A) Crude-analysis. (B) Propensity score matching analysis. DAPT, dual antiplatelet therapy; MACCE, major adverse cardiovascular and cerebrovascular events. 


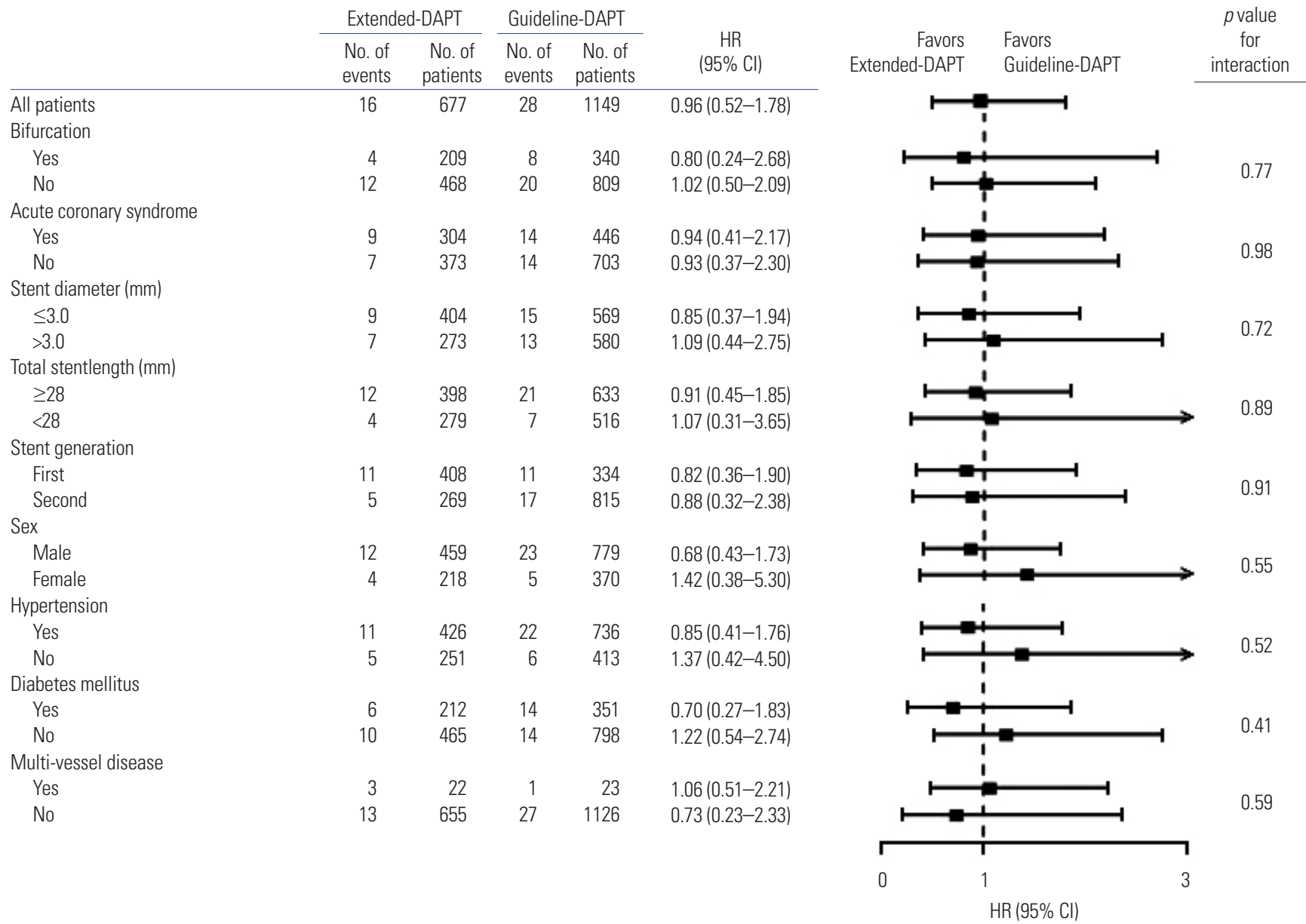

Fig. 3. Subgroup analyses. HRs for NACE from Cox regression models investigating extended-DAPT versus guideline-DAPT are shown for patient subgroups. DAPT, dual antiplatelet therapy; HR, hazard ratio; $\mathrm{Cl}$, confidence interval; NACE, net adverse clinical events.

The type of DES was also a significant determining factor for the extended use of DAPT. Some registry data have indicated a continually increasing risk of ST several years after first-generation DES implantation. ${ }^{22}$ Currently available registry data suggest a lower rate of ST in patients who received second-generation DESs compared to those who received firstgeneration DESs. ${ }^{23}$ In the patients who received first-generation DESs in this study, there was a lower rate of MACCE in the extended-DAPT group compared to the guideline-DAPT group. A previous study (ARTIC-Generation; Clinical Outcome of First- vs. Second-Generation DES According to DAPT Duration) also indicated worse clinical outcomes with first-generation DESs compared to second-generation DESs, a difference that appeared to persist even with prolonged DAPT (6.2\% vs. 2.6\%; HR, 2.31; 95\% CI, 1.31-4.07; $p=0.004) .{ }^{24}$ In the current study, extended DAPT use led to a significant reduction in MAC$\mathrm{CE}$ in patients who were treated with first-generation DESs. Based on the results of this study and other studies, extended DAPT could be carefully considered for patients treated with first-generation DESs.

There are several concerns regarding extended DAPT, such as increased major bleeding [ARCTIC-Interruption (Dual-An- tiplatelet Treatment beyond 1 Year after Drug-Eluting Stent Implantation): 12 -month vs. $18-30$ month DAPT, $<0.5 \%$ vs. $1 \%$, $p=0.073$; DAPT: 12 -month vs. 30 -month DAPT, $1.6 \%$ vs. $2.5 \%$, $p=0.001$; PRODIGY (Prolonging Dual Antiplatelet Treatment After Grading Stent-Induced Intimal Hyperplasia Study): 24month vs. 6 -month DAPT, $7.4 \%$ vs. $3.5 \%, p=0.0002]]^{3,13,25}$

However, in the current study, extended DAPT did not significantly increase the risk of major bleeding after propensity matching ( $2.1 \%$ vs. $1.2 \%, p=0.07)$. Therefore, extended DAPT could be used in patients at high thrombotic risk without excessively increasing the risk of major bleeding. In clinical practice, the duration of DAPT may be tailored after careful evaluation of both bleeding and ischemic risk, while taking into consideration the clinical presentation, lesion complexity, and procedural factors.

The strength of this study lies in its use of population-based, real-world registries. However, this study had several limitations. First, this was a retrospective analysis of registry data. Second, since the current study included only patients who did not experience a major event during 3 years after PCI and were compliant with the designated therapy, the study results were only relevant to patients who achieved that milestone. In 
interpreting the results of the current study, the group of subjects should not be expanded, and the results should be interpreted with caution. Third, neither medication adherence nor tolerability was evaluated. Fourth, as the patients enrolled in this study were event-free for 3 years, there was a possibility that the patients at low risk of bleeding or cardiovascular events were enrolled. Fifth, patients who had used DAPT for $>3$ years were classified into the extended-DAPT group, but this classification was arbitrary. Careful attention would be needed for interpretation of the current results. Notwithstanding, $86.4 \%$ of the patients in the extended-DAPT group actually used DAPT for more than 5 years. Sixth, the classification of the guideline, intermediate, and extended-DAPT groups may be an arbitrary classification. The guideline-DAPT group was developed for comparison with the extended-DAPT group, and was classified according to the duration of DAPT use. Seventh, we could not achieve perfect matching or control of all of the confounding variables, even though we conducted propensity score matching analysis to reduce bias. However, we were able to exclude all of the extremes in each group. Finally, this study only included thienopyridine P2Y12 inhibitors. Therefore, additional studies comparing the extended DAPT using new antiplatelet agents, such as ticagrelor and prasugrel, with standard DAPT should be performed to further guide clinical practice.

In conclusion, the significant predictors for the use of extended DAPT for more than 3 years were ACS, bifurcation, small stent diameter, long length of DES, and first-generation DES in the real world. In patients undergoing PCI, indefinite use of DAPT did not show superiority in reducing the clinical events compared to the guideline-DAPT. Moreover, major bleeding rates were similar. Notably, however, in patients who were treated with first-generation DESs, extended-DAPT was associated with significantly lower MACCE rates compared to guidelineDAPT.

\section{ACKNOWLEDGEMENTS}

This study was supported by a grant from the Korea Healthcare Technology Research and Development Project, Ministry for Health and Welfare, Republic of Korea (Nos. A085136 and HI15 C1277); the Mid-Career Researcher Program through an NRF grant funded by the MEST, Republic of Korea (No. 2015R1A2 A2A01002731); and the Cardiovascular Research Center, Seoul, Republic of Korea.

\section{AUTHOR CONTRIBUTIONS}

Conceptualization: Byeong-Keuk Kim. Data curation: Oh-Hyun Lee and Byeong-Keuk Kim. Formal analysis: Oh-Hyun Lee. Funding acquisition: Byeong-Keuk Kim. Investigation: Oh-Hyun Lee and ByeongKeuk Kim. Methodology: Oh-Hyun Lee and Byeong-Keuk Kim. Project administration: Byeong-Keuk Kim. Resources: Oh-Hyun Lee and Byeong-Keuk Kim. Software: Oh-Hyun Lee. Supervision: ByeongKeuk Kim. Validation: Oh-Hyun Lee and Byeong-Keuk Kim. Visual- ization: Oh-Hyun Lee. Writing_original draft: Oh-Hyun Lee, ByeongKeuk Kim. Writing_-review \& editing: Sung-Jin Hong, Seunghwan Kim, Chul-Min Ahn, Dong-Ho Shin, Jung-Sun Kim, Tae Soo Kang, Young-Guk Ko, Donghoon Choi, Myeong-Ki Hong, and Yangsoo Jang. Approval of final manuscript: all authors.

\section{ORCID iDs}

Oh-Hyun Lee https://orcid.org/0000-0001-7070-7720

Byeong-Keuk Kim https://orcid.org/0000-0003-2493-066X

Sung-Jin Hong https://orcid.org/0000-0003-4893-039X

Seunghwan Kim https://orcid.org/0000-0001-6783-3849

Chul-Min Ahn https://orcid.org/0000-0002-7071-4370

Dong-Ho Shin https://orcid.org/0000-0002-7874-5542

Jung-Sun Kim https://orcid.org/0000-0003-2263-3274

Tae Soo Kang https://orcid.org/0000-0002-4298-6272

Young-Guk Ko https://orcid.org/0000-0001-7748-5788

Donghoon Choi https://orcid.org/0000-0002-2009-9760

Myeong-Ki Hong https://orcid.org/0000-0002-2090-2031

Yangsoo Jang https://orcid.org/0000-0002-2169-3112

\section{REFERENCES}

1. Gargiulo G, Valgimigli M, Capodanno D, Bittl JA. State of the art: duration of dual antiplatelet therapy after percutaneous coronary intervention and coronary stent implantation-past, present and future perspectives. EuroIntervention 2017;13:717-33.

2. Levine GN, Bates ER, Bittl JA, Brindis RG, Fihn SD, Fleisher LA, et al. 2016 ACC/AHA Guideline Focused Update on Duration of Dual Antiplatelet Therapy in Patients With Coronary Artery Disease: A Report of the American College of Cardiology/American Heart Association Task Force on Clinical Practice Guidelines: An Update of the 2011 ACCF/AHA/SCAI Guideline for Percutaneous Coronary Intervention, 2011 ACCF/AHA Guideline for Coronary Artery Bypass Graft Surgery, 2012 ACC/AHA/ACP/AATS/PCNA/ SCAI/STS Guideline for the Diagnosis and Management of Patients With Stable Ischemic Heart Disease, 2013 ACCF/AHA Guideline for the Management of ST-Elevation Myocardial Infarction, 2014 AHA/ACC Guideline for the Management of Patients With Non-ST-Elevation Acute Coronary Syndromes, and 2014 ACC/AHA Guideline on Perioperative Cardiovascular Evaluation and Management of Patients Undergoing Noncardiac Surgery. Circulation 2016;134:e123-55.

3. Mauri L, Kereiakes DJ, Yeh RW, Driscoll-Shempp P, Cutlip DE, Steg PG, et al. Twelve or 30 months of dual antiplatelet therapy after drug-eluting stents. N Engl J Med 2014;371:2155-66.

4. Kim JS, Lee BH, Ko YG, Choi D, Jang Y, Min PK, et al. Comparison of sirolimus-eluting stent and paclitaxel-eluting stent for longterm cardiac adverse events in diabetic patients: the Korean Multicenter Angioplasty Team (KOMATE) registry. Catheter Cardiovasc Interv 2008;72:601-7.

5. Cutlip DE, Windecker S, Mehran R, Boam A, Cohen DJ, van Es GA, et al. Clinical end points in coronary stent trials: a case for standardized definitions. Circulation 2007;115:2344-51.

6. Thygesen K, Alpert JS, Jaffe AS, Simoons ML, Chaitman BR, White $\mathrm{HD}$, et al. Third universal definition of myocardial infarction. Circulation 2012;126:2020-35.

7. Sacco RL, Kasner SE, Broderick JP, Caplan LR, Connors JJ, Culebras A, et al. An updated definition of stroke for the 21st century: a statement for healthcare professionals from the American Heart Association/American Stroke Association. Stroke 2013;44:2064-89.

8. Hicks KA, Stockbridge NL, Targum SL, Temple RJ. Bleeding aca- 
demic research consortium consensus report: the food and drug administration perspective. Circulation 2011;123:2664-5.

9. Ponikowski P, Voors AA, Anker SD, Bueno H, Cleland JGF, Coats AJS, et al. 2016 ESC guidelines for the diagnosis and treatment of acute and chronic heart failure: the task force for the diagnosis and treatment of acute and chronic heart failure of the european society of cardiology (ESC). Developed with the special contribution of the heart failure association (HFA) of the ESC. Eur J Heart Fail 2016;18:891-975.

10. Thomas M, Hildick-Smith D, Louvard Y, Albiero R, Darremont O, Stankovic G, et al. Percutaneous coronary intervention for bifurcation disease. A consensus view from the first meeting of the European Bifurcation Club. EuroIntervention 2006;2:149-53.

11. Yeh RW, Secemsky EA, Kereiakes DJ, Normand SLT, Gershlick AH, Cohen DJ, et al. Development and validation of a prediction rule for benefit and harm of dual antiplatelet therapy beyond 1 year after percutaneous coronary intervention. JAMA 2016;315:1735-49.

12. Kim BK, Hong MK, Shin DH, Nam CM, Kim JS, Ko YG, et al. A new strategy for discontinuation of dual antiplatelet therapy: the RESET Trial (REal Safety and Efficacy of 3-month dual antiplatelet Therapy following Endeavor zotarolimus-eluting stent implantation). J Am Coll Cardiol 2012;60:1340-8.

13. Collet JP, Silvain J, Barthélémy O, Rangé G, Cayla G, Belle EV, et al. Dual-antiplatelet treatment beyond 1 year after drug-eluting stent implantation (ARCTIC-Interruption): a randomised trial. Lancet 2014;384:1577-85.

14. Palmerini T, Benedetto U, Bacchi-Reggiani L, Della Riva D, Biondi-Zoccai G, Feres F, et al. Mortality in patients treated with extended duration dual antiplatelet therapy after drug-eluting stent implantation: a pairwise and Bayesian network meta-analysis of randomised trials. Lancet 2015;385:2371-82.

15. Mehran R, Baber U, Steg PG, Ariti C, Weisz G, Witzenbichler B, et al. Cessation of dual antiplatelet treatment and cardiac events after percutaneous coronary intervention (PARIS): 2 year results from a prospective observational study. Lancet 2013;382:1714-22.

16. Baber U, Mehran R, Giustino G, Cohen DJ, Henry TD, Sartori S, et al. Coronary thrombosis and major bleeding after PCI with drugeluting stents: risk scores from PARIS. J Am Coll Cardiol 2016;67:
2224-34.

17. Costa F, van Klaveren D, James S, Heg D, Räber L, Feres F, et al. Derivation and validation of the predicting bleeding complications in patients undergoing stent implantation and subsequent dual antiplatelet therapy (PRECISE-DAPT) score: a pooled analysis of individual-patient datasets from clinical trials. Lancet 2017;389:1025-34.

18. Yeh RW, Kereiakes DJ, Steg PG, Windecker S, Rinaldi MJ, Gershlick AH, et al. Benefits and risks of extended duration dual antiplatelet therapy after PCI in patients with and without acute myocardial infarction. J Am Coll Cardiol 2015;65:2211-21.

19. Cutlip DE, Baim DS, Ho KK, Popma JJ, Lansky AJ, Cohen DJ, et al. Stent thrombosis in the modern era: a pooled analysis of multicenter coronary stent clinical trials. Circulation 2001;103:1967-71.

20. Cook S, Wenaweser P, Togni M, Billinger M, Morger C, Seiler C, et al. Incomplete stent apposition and very late stent thrombosis after drug-eluting stent implantation. Circulation 2007;115:2426-34.

21. Jang WJ, Ahn SG, Song YB, Choi SH, Chun WJ, Oh JH, et al. Benefit of prolonged dual antiplatelet therapy after implantation of drug-eluting stent for coronary bifurcation lesions: results from the coronary bifurcation stenting registry II. Circ Cardiovasc Interv 2018;11:e005849.

22. Daemen J, Wenaweser P, Tsuchida K, Abrecht L, Vaina S, Morger C, et al. Early and late coronary stent thrombosis of sirolimus-eluting and paclitaxel-eluting stents in routine clinical practice: data from a large two-institutional cohort study. Lancet 2007;369:667-78.

23. Lagerqvist B, Carlsson J, Fröbert O, Lindbäck J, Scherstén F, Stenestrand U, et al. Stent thrombosis in Sweden: a report from the Swedish Coronary Angiography and Angioplasty Registry. Circ Cardiovasc Interv 2009;2:401-8.

24. Collet JP, Silvain J, Kerneis M, Cuisset T, Meneveau N, Boueri Z, et al. Clinical outcome of first- vs second-generation DES according to DAPT duration: results of ARCTIC-generation. Clin Cardiol 2016;39:192-200.

25. Valgimigli M, Campo G, Monti M, Vranckx P, Percoco G, Tumscitz $\mathrm{C}$, et al. Short- versus long-term duration of dual-antiplatelet therapy after coronary stenting: a randomized multicenter trial. Circulation 2012;125:2015-26. 